\title{
O perfil dos cuidadores de pacientes pediátricos com fibrose cística
}

\author{
The profile of caregivers to pediatric patients with cystic fibrosis
}

Stella Pegoraro Alves ${ }^{1}$

Denise Bueno ${ }^{1}$
${ }^{1}$ Programa de PósGraduação em Assistência Farmacêutica, Universidade Federal do Rio Grande do Sul. Av. Paulo Gama 110, Farroupilha. 90040-060 Porto Alegre RS Brasil. stellape@gmail.com

\begin{abstract}
The scope of this study was to establish the profile of caregivers of pediatric patients diagnosed with Cystic Fibrosis (CF). It was a cross-sectional, descriptive and prospective study in which the caregivers of fibrocystic patients were interviewed during pharmaceutical consultation in a reference center of a University Hospital in southern Brazil. General information was obtained about the caregivers and about their understanding of the disease, drug consumption and dynamics of treatment at home and at school. Seventy-five caregivers were interviewed. Most of them were female, 37.3 years old on average, mothers of the patients who did not work outside the home. Seventy-one caregivers declared difficulties in drug acquisition and patient support associations were highlighted as the main alternative to avoid the interruption of treatment. Another fact observed was the overload of the caregiving process on the shoulders of only one caregiver resulting in social and economic impacts and changes to the family's daily routine. This fact emphasizes the need of intervention by a qualified multidisciplinary team to identify and alleviate difficulties, investing in interpersonal relations and administering care.
\end{abstract}

Key words Cystic fibrosis, Chronic disease, Child, Caregivers, Pharmaceutical services
Resumo O objetivo desse estudo foi identificar o perfil do cuidador principal de pacientes pediátricos com Fibrose Cística (FC). Estudo transversal, descritivo e prospectivo, no qual foi realizado, durante consulta farmacêutica, entrevista com cuidadores de pacientes fibrocísticos acompanhados em um centro de referência de um Hospital Universitário do Sul do Brasil. Foram obtidas informações gerais sobre os cuidadores, sobre o entendimento da doença, os medicamentos em uso do paciente e a dinâmica do tratamento em domicílio e na escola. Foram entrevistados 75 cuidadores. Maioria do sexo feminino, com média de idade de 37,3 anos, mãe do paciente e que não trabalhava fora do domicílio. Sessenta e um cuidadores referiram dificuldade de aquisição de medicamentos e as associações de apoio ao paciente foram a principal alternativa para a não interrupção do tratamento. A sobrecarga do processo do cuidar que recai em um único cuidador, gerando impacto social e econômico e alterando o cotidiano familiar foi outro dado observado. Foi constatada a necessidade da intervenção de equipe multidisciplinar capacitada na identificação das dificuldades apresentadas, investindo nas relações interpessoais e efetivando a assistência.

Palavras-chave Fibrose cística, Doença crônica, Criança, Cuidadores, Assistência farmacêutica 


\section{Introdução}

A Fibrose Cística (FC), ou mucoviscidose, é uma doença hereditária, autossômica recessiva, letal, muito comum entre caucasianos. A doença acomete diversos sistemas, principalmente respiratório, gastrointestinal e reprodutor; além do fígado e células secretoras. Essas manifestações podem surgir ao longo da vida e variar de paciente para paciente ${ }^{1,2}$.

A FC é uma doença de caráter multissistêmico, crônico e progressivo que necessita de um cuidado complexo e contínuo para manutenção e promoção da melhoria da qualidade de vida do paciente. Essa atenção em tempo integral, principalmente quando falamos em crianças e adolescentes, faz com que os cuidadores necessitem conhecer a doença e a terapêutica possibilitando a assistência desejada ao fibrocístico ${ }^{3}$.

Dados de 2013, do Registro Brasileiro de Fibrose Cística (REBRAFC), notificaram quase 3000 pacientes com diagnóstico de FC. Os estados do Sudeste e Sul do país possuem o maior número de pacientes registrados. O Rio Grande do Sul é o segundo Estado brasileiro com mais pacientes: 348 nascidos e 378 pacientes são acompanhados em seus três centros de referencia para a doença $a^{4}$.

A situação de doença, principalmente de característica crônica, como a FC, exige por parte de todos os membros que fazem parte da vida do paciente, a sua participação no processo de adaptação e de compreensão à uma nova realidade no cotidiano da criança. E cada família possui maneiras próprias de administrar essas situações diante da vulnerabilidade da saúde da criança ${ }^{5}$.

A sobrevida desses pacientes é dependente de diversos fatores, tais como necessidade de diagnóstico precoce, manejo multidisciplinar, adequado aporte nutricional e de avanços em relação ao tratamento medicamentoso ${ }^{2,6}$. Em alguns países, a sobrevida é de 40 anos, porém estudos afirmam que a mesma possa se estender para 50 anos de idade aos nascidos com a doença nos dias de hoje $\mathrm{e}^{7}$.

Mesmo sabendo que essa nova situação afeta as famílias como um todo, a responsabilidade principal sobre o cuidado do paciente geralmente recai sobre um único familiar, que denominamos o cuidador principal. Essa é a pessoa responsável no auxílio do paciente no seu dia-a-dia, seja na administração dos medicamentos, na realização de procedimentos como a fisioterapia e atividades físicas e no acompanhamento das consultas e das internações ${ }^{8,9}$.
O cuidador principal deve ser o maior aliado dos membros da equipe multiprofissional que presta assistência ao paciente?. Para isso, é imprescindível que os profissionais de saúde conheçam a família em todos os aspectos: sociais, emocionais e financeiros ${ }^{3,6}$.

Quando a equipe conhece a realidade do paciente e em qual contexto ele está inserido, ela realiza as intervenções necessárias para a melhora da clínica do paciente, do controle dos sintomas, do conhecimento da doença e do regime terapêutico, promovendo a adesão e a participação do apoio familiar. Quando a família passa a participar do tratamento e das decisões sobre os cuidados de saúde do paciente, o enfrentamento em relação ao impacto da doença e da terapêutica é facilitado ${ }^{10}$.

Este estudo teve como objetivo identificar o perfil do cuidador principal de pacientes pediátricos com FC, assim como os caminhos percorridos e as dificuldades enfrentadas pelos mesmos perante o tratamento.

\section{Métodos}

Estudo transversal, descritivo e prospectivo, realizado com cuidadores de pacientes com FC acompanhados no ambulatório de pneumologia infantil de um Hospital Universitário do Sul do Brasil. Os pacientes frequentam o ambulatório com periodicidade mensal ou bimestral. $\mathrm{O}$ ambulatório deste serviço é um dos três centros de referência de FC no Estado, para controle da doença e revisão do tratamento. Estes pacientes são assistidos por uma equipe multiprofissional composta por médicos (pneumologistas e psiquiatras), enfermeiros, nutricionistas, fisioterapeutas, educadores físicos, psicólogos, assistentes sociais e farmacêuticos.

As consultas foram previamente agendadas e os atendimentos realizados conforme ordem de chegada. Os cuidadores foram convidados a participar do estudo de forma aleatória, durante consulta farmacêutica no ambulatório, nos períodos de dezembro de 2014 a maio de 2015. Foram considerados como critério de exclusão os pacientes que estavam desacompanhados e/ ou que não possuíam cuidador (responsabilidade do tratamento era do próprio paciente). Os cuidadores e pacientes que não compareceram às consultas ou os que não foram atendidos pelo farmacêutico não participaram do estudo.

Foram coletadas informações gerais e clínicas dos pacientes (sexo, idade e presença de compli- 
cações pulmonares, de insuficiência pancreática e de doença e/ou comorbidades) obtidas através do prontuário eletrônico do paciente. $\mathrm{E}$ as informações sobre os cuidadores, como sexo, parentesco, idade, escolaridade, profissão e/ou ocupação, utilização de tratamento medicamentoso contínuo e entendimento da FC; informações sobre os medicamentos em uso do paciente como a indicação terapêutica e aquisição; informações da dinâmica do tratamento em domicílio, como dificuldades, responsabilidades e revezamento do cuidado; além de informações referentes ao envolvimento da escola (total, parcial, nenhum) para os pacientes que frequentavam, foram obtidas através da entrevista com o farmacêutico.

Em relação ao entendimento da doença, foi questionado ao cuidador qual conhecimento sobre a FC. O que ele entendia sobre a FC, quais eram os sintomas, as consequências e curso da doença e a importância da realização correta do tratamento. As respostas obtidas foram classificadas em entendimento bom, regular e ruim.

Os dados quantitativos obtidos no estudo foram armazenados e analisados utilizando o programa SPSS 22.0. As variáveis contínuas foram descritas por médias e desvio padrão ou por mediana e intervalo interquartil quando distribuição assimétrica. Utilizaram-se as frequências absolutas e relativas para descrever as variáveis categóricas e nas associações entre elas foi utilizado o teste qui-quadrado. Para variáveis quantitativas empregou-se o t de Student. Os valores foram considerados estatisticamente significativos quando o valor de $\mathrm{p}<0,05$.

Os cuidadores que participaram do estudo assinaram o termo de consentimento livre e esclarecido, assim como os pacientes acima de 12 anos, o termo de assentimento. $\mathrm{O}$ estudo foi aprovado pelo Comitê de Ética em Pesquisa, do Grupo de Pesquisa e Pós Graduação da instituição na qual o estudo foi realizado.

\section{Resultados}

Dos 78 convidados para a pesquisa, três pacientes relataram que não possuíam cuidador e duas famílias tinham dois filhos com diagnóstico de FC. Devido a isso, foram totalizados 75 entrevistados.

A maioria dos cuidadores $(98,7 \%)$ foi do sexo feminino e $96 \%$ dos casos configuraram-se na figura materna. Um cuidador foi o pai e duas cuidadoras, as avós maternas dos pacientes. Apenas 14 entrevistados $(17,9 \%)$ informaram revezar os cuidados. Em 71,4\%, a divisão do cuidado era realizada com o pai da criança. As informações gerais dos pacientes foram descritas na Tabela 1 .

A idade dos cuidadores variou de 20 a 64 anos, sendo a média de 37,3 anos (mediana 36 anos). Os dados não apresentaram relação entre a idade dos cuidadores e o entendimento da doença.

Todos os entrevistados eram alfabetizados e a maioria $(29,3 \%)$ possuía o ensino médio completo. Do total, 51\% não trabalhavam fora do domicilio, sendo donas de casa, aposentados ou realizavam atividades extras no próprio lar, como costureiras. As profissões ou ocupações citadas pelos demais entrevistados foram agricultor, micro ou pequeno empresário, cabeleireira, recepcionista, professora, entre outras. Houve associação entre o cuidador que trabalha fora do domicilio e a presença de complicações pulmonares $(p=0,047)$ e de doenças e/ou comorbidades associadas à $\mathrm{FC}(\mathrm{p}=0,027)$.

Mais da metade dos cuidadores (64\%) demonstrou possuir bom entendimento sobre a FC, 25,3\% um entendimento regular e 10,7\% um entendimento ruim da doença. Os dados não mostraram relação entre o entendimento da doença e a idade e escolaridade dos cuidadores.

Tabela 1. Características gerais dos pacientes $(n=75)$.

\begin{tabular}{lc}
\hline \multicolumn{1}{c}{ Características } & Valores \\
\hline Sexo & $41(54,7 \%)$ \\
Feminino & $34(45,3 \%)$ \\
Masculino & $8,6 \pm 5,5$ anos \\
Idade (anos) & \\
Procedência & $50(66,7 \%)$ \\
Interior & $13(17,3 \%)$ \\
Região Metropolitana & $8(10,7 \%)$ \\
Capital & $4(5,3 \%)$ \\
Outro Estado & \\
Complicações Pulmonares & $34(45,3 \%)$ \\
Não & $41(54,7 \%)$ \\
Sim & \\
Insuficiência Pancreática & $7(9,3 \%)$ \\
Não & $68(90,7 \%)$ \\
Sim & \\
Doença e/ou comorbidades & $45(60 \%)$ \\
Não & $30(40 \%)$ \\
Sim & \\
Frequentava escola & $53(70,7 \%)$ \\
Sim & $22(29,3 \%)$ \\
Não &
\end{tabular}


Em 73,3\% dos casos, os cuidadores eram os principais responsáveis tanto pelo tratamento medicamentoso, quanto pela realização dos procedimentos, como a fisioterapia em domicílio. Em 26,7\% essa responsabilidade era compartilhada entre cuidador e paciente.

A frequência dos pacientes acompanhados exclusivamente nesse ambulatório foi de 56,6\%. Os demais eram vinculados a outros locais e serviços de saúde, como consultórios médicos privados e unidades básicas de saúde. $\mathrm{O}$ acompanhamento do tratamento extra-ambulatorial apresentou associação ao bom entendimento da doença $(\mathrm{p}=0,007)$.

Sobre os medicamentos, a média foi de 6,5 medicamentos prescritos por paciente de um total de 485. Desses, os entrevistados souberam relatar a utilização correta (indicação terapêutica) de mais de $80 \%$ deles. Cerca de $54 \%$ dos medicamentos foram adquiridos através da Secretaria Estadual de Saúde (SES), seguidos de farmácias privadas $(25,9 \%)$ e de UBS $(6,9 \%)$.

Sessenta e um entrevistados referiram dificuldade de aquisição de um ou mais medicamentos, totalizando 97 medicamentos. Os mais citados foram os polivitamínicos, medicamentos importados pela SES.

Desses, 74 medicamentos foram adquiridos por outros meios. As associações de apoio ao paciente com FC foram a forma de alternativa encontrada pelo paciente para adquirir seu medicamento em $22,4 \%$ dos casos estudados.

Foi realizada a substituição da prescrição do medicamento em falta por outro, em $17,2 \%$ dos casos; $13,6 \%$ dos cuidadores informaram possuir sobras de medicamentos em casa. Quanto a doações, 10,2\% recebiam doações de outros pacientes. Os cuidadores em 9,1\% dos casos informaram comprar o medicamento quando disponível na rede privada. Em $24,7 \%$ das situações, os pacientes permaneceram sem o medicamento.

As dificuldades no tratamento, além da aquisição dos medicamentos, foram relatadas por $32 \%$ dos entrevistados: $11,5 \%$ informaram dificuldade na administração dos medicamentos, $10,3 \%$ na realização da fisioterapia, e outros $10,2 \%$ relataram outras dificuldades como na alimentação do paciente, realização de atividades físicas e renovação das receitas.

Todos os cuidadores moravam com o paciente. A quantidade total de moradores variou de 2 a 7 moradores (mediana 3 moradores). Em 32\% desses lares, algum familiar, além do próprio paciente, realizava algum tratamento medicamentoso contínuo. Hipertensão, Diabetes, Asma, doenças cardíacas e doenças psiquiátricas foram algumas das doenças relatadas pelos entrevistados. A realização de tratamento medicamentoso de algum familiar apresentou associação com a presença de complicações pulmonares entre os pacientes $(\mathrm{p}=0,007)$.

Para os pacientes que frequentavam escola, $67,9 \%$ dos cuidadores consideraram que a mesma possuía envolvimento total no tratamento do paciente, possuía conhecimento do diagnóstico e auxiliavam o paciente, seja nos medicamentos a serem utilizados na escola, nas tarefas a serem realizadas durante internações e nas atividades físicas próprias para o paciente, entre outras situações; $26,4 \%$ informaram que a escola tinha conhecimento do diagnóstico mas que não havia auxilio da mesma no tratamento, considerando assim, o envolvimento parcial e 5,7\% informaram que a escola não possuia nenhum envolvimento com o paciente a respeito da doença e dos cuidados necessários.

\section{Discussão}

Os cuidadores foram na maioria do sexo feminino, com média de 37,3 anos de idade. Dados de acordo com a literatura a respeito de cuidadores de crianças e adolescentes com a mesma doença ${ }^{11-13}$. O mesmo pode ser observado em estudos de pacientes com outras doenças crônicas ${ }^{8,14}$.

A maioria das famílias foi proveniente do interior do Estado. Informação observada em outros estudos ${ }^{3,15}$. O tratamento multidisciplinar especializado para FC, em grande parte do país, não é disponibilizado nas cidades de origem dos pacientes, sendo a maioria dos grandes centros de referência para a doença, distribuído somente nas capitais brasileiras. Com isso, as famílias necessitam manter uma rotina desgastante de viagens para a continuidade da assistência integral à criança $\mathrm{a}^{15}$.

Para quase todos os pacientes, com exceção de três adolescentes, a mãe era a principal cuidadora. O cuidado com o paciente fibrocístico exige, muitas vezes, uma alteração do cotidiano da família. Estudos relatam, através da fala dos familiares, que a sobrecarga da doença crônica recai sobre a figura materna, que geralmente é a principal cuidadora, tanto nos cuidados e procedimentos realizados, como na evolução do paciente ${ }^{3,13}$. Além dos cuidados diários com administração de medicamentos e a realização de procedimentos necessários para melhorar a qualidade de vida dessas crianças, as internações 
frequentes por exacerbação da FC e para antibioticoterapia e/ou outros medicamentos via parenteral, faz com que os cuidadores se afastem das suas atividades corriqueiras, tanto pelo cansaço quanto pela falta de tempo ${ }^{11,14}$.

No presente estudo, $51 \%$ dos cuidadores não trabalhavam fora. Desses, alguns deixaram de trabalhar para ficar em casa devido às necessidades e dependência da criança. Essa atitude pode trazer consequências para a família como um todo, uma vez que o cuidador que deixa de trabalhar não contribui mais financeiramente, impactando negativamente na condição socioeconômica da família. Podendo refletir, por exemplo, na dificuldade de aquisição de medicamentos, materiais, suplementação alimentar e procedimentos de alto custo 3 .

Os dados apresentaram relação direta entre o cuidador que trabalha fora e a presença de complicações pulmonares e de comorbidades. Tavares et al. ${ }^{11}$ reforçam que os cuidadores que possuem a tripla jornada de se dedicar a uma profissão ou ocupação, trabalhar com as atividades do lar e de ser cuidador, podem contribuir para a potencialização das dificuldades já enfrentadas, entre elas a piora do estado clínico do paciente.

Frequentemente, à medida que a doença do paciente se agrava, o cuidador negligencia o cuidado com a própria saúde ${ }^{16}$. O que pode ser observado nesse estudo, no qual, de $32 \%$ das famílias que possuem tratamento medicamentoso contínuo, em $46 \%$, o tratamento era realizado pelo principal cuidador ( $91 \%$ a mãe do paciente). É sabido que a presença da FC afeta não só a criança e o adolescente a ser cuidado, mas também a saúde do seu cuidador ${ }^{17}$.

Os cuidadores souberam informar satisfatoriamente sobre a FC, assim como seus sintomas, consequências e importância da realização correta do tratamento na maioria dos casos. O paciente com acompanhamento exclusivamente ambulatorial, em algumas situações, diminui o contato com outros profissionais e serviços, principalmente os acerca da sua residência, que podem estar prestando uma assistência mais próxima e pontual. Porém é necessária a troca de saberes e experiências entre as equipes que prestam assistência a esses pacientes assim como entre os profissionais e pacientes e seus cuidadores, buscando melhoria das práticas de cuidado, preservando as características do contexto que essas famílias estão inseridas e ampliando o conhecimento da doença e do cuidado ${ }^{10}$.

Vinte cuidadores relataram que dividiam a responsabilidade da administração dos medica- mentos e a realização de alguns procedimentos como fisioterapia, com o próprio paciente. Para isso, contar com uma equipe multiprofissional capacitada, que possa estar atuando nessa fase crucial, para a promoção da autonomia e do autocuidado dos pacientes, assim como para continuidade correta do tratamento é imprescindível $^{18}$. Outro estudo aborda a importância do próprio paciente estar preocupado com seu autocuidado, seja na busca de sua independência ou como estratégia de controle de suas atividades para melhor planejamento do cotidiano e minimizando impacto da doença, melhorando o processo de socialização, especialmente no ambiente escolar ${ }^{19}$.

Pizzignacco et al. ${ }^{20}$ reforçam que os profissionais de saúde possam estar auxiliando, principalmente nas que já possuam crianças e/ou adolescentes com FC, com ações educativas e visitas em escolas. Políticas de saúde e educação que capacitam os profissionais de saúde, como trabalhar as questões relacionadas ao estigma da doença e a adesão ao tratamento, pode-se detectar os problemas, e estabelecer maior articulação entre esses dois setores para maior integralidade da assistência a esses pacientes e famílias ${ }^{19}$.

Os cuidadores souberam informar a indicação terapêutica dos medicamentos em uso pelas suas crianças em $82,5 \%$ dos casos. Dado semelhante foi encontrado em outro estudo, no mesmo hospital de ensino, mas com diferentes sujeitos de pesquisa ${ }^{21}$.

Cerca de $80 \%$ dos cuidadores informaram dificuldade de aquisição de medicamentos. O cuidador, muitas vezes, desconhece a forma de adquirir os medicamentos e tratamento da doença, necessitando que o mesmo percorra diversos itinerários terapêuticos na busca da garantia da qualidade de vida desses pacientes. A partir das redes de apoio e das trocas de experiências que as famílias desenvolvem meios para superar as dificuldades encontradas na assistência ${ }^{3}$.

A alternativa mais evidente para aquisição de medicamentos foi a busca às associações de FC. Entender as necessidades das famílias com portadores de FC reforçou a importância da divulgação desses serviços que ampliam a rede de apoio. Essas associações, criadas no Brasil a partir do final da década de 70, são instrumentos de promoção da saúde, que, através de serviços e profissionais de saúde, trabalham para que o sofrimento do viver com FC seja o mínimo possível para os pacientes, cuidadores e família ${ }^{12,22}$.

Outro papel importante, não somente das associações como também dos próprios centros de 
referência, é a divulgação da doença. Estudo relata que mães de pacientes informaram que a falta de conhecimento geral, o estigma e o preconceito da população em relação à FC acabam limitando a sua rede de apoio ${ }^{11}$.

As dificuldades em relação aos medicamentos, tanto na aquisição quanto na administração e às tarefas que envolvem as demais etapas do tratamento, como realização da fisioterapia, a alimentação do paciente e a realização das atividades físicas foram alguns dos entraves identificados. A sobrecarga do cuidar desse paciente crônico que recai, em especial, sobre a figura materna desse paciente, impacta diretamente nos aspectos sociais e econômicos, não somente dessa mãe como no de toda a rede de apoio no qual o paciente está inserido. Essas situações evidenciam a necessidade de profissionais da saúde qualificados e preparados para atuarem no apoio a essas famílias, tanto na orientação da realização do cuidado quanto na escuta qualificada, identificando as dificuldades apresentadas, investindo nas relações interpessoais e efetivando a assistência.

\section{Colaboradores}

O manuscrito foi produzido, escrito, revisado e aprovado por SP Alves e D Bueno.

\section{Agradecimentos}

Fundação de Amparo à Pesquisa do Estado do Rio Grande do Sul (FAPERGS) e Equipe Multiprofissional do Ambulatório de Pneumologia Infantil do Hospital de Clínicas de Porto Alegre. 


\section{Referências}

1. O'Sullivan BP, Freedman SD. Cystic Fibrosis. Lancet 2009; 373(9678):1891-1904.

2. Ribeiro JD, Ribeiro MA, Ribeiro AF. Controversies in cystic fibrosis--from pediatrician to specialist. J Pediatr (Rio J) 2002; 78(Supl. 2):S171-S186.

3. Furtado MC, Lima RA. Daily routine of families with cystic fibrosis children: subsides for pediatric nursing. Rev Lat Am Enfermagem 2003; 11(1):66-73.

4. Grupo Brasileiro de Estudos de Fibrose Cística (GBEFC). Registro Brasileiro de Fibrose Cística (REBRAFC). [Internet]. [acessado 2016 jan 12]. Disponível em: http://conteudosportal.com.br/GBEFC/wp-content/ uploads/2016/03/REBRAFC_2013.pdf

5. Silva FM, Correa I. Doença crônica na infância: vivência do familiar na hospitalização da criança. REME Rev Min Enf 2006; 10(1):18-23.

6. Scattolin I, Beier S, Blacher J, Laurent MC, Santos MIS. Desenvolvimento da atenção integral à criança com fibrose cística. In: Carvalho PRA, Ceccim RB, organizadores. Criança hospitalizada: atenção integral como escuta à vida. Porto Alegre: UFRGS; 1997. p. 90-95.

7. Bell SC, De Boeck K, Amaral MD. New pharmacological approaches for cystic fibrosis: promises, progress, pitfalls. Pharmacol Ther 2015; 145:19-34.

8. Araújo LZ, Araújo CZ, Souto AK, Oliveira MS. The main caregiver of oncologic terminally ill patient, repercussions of this task. Rev Bras Enferm 2009; 62(1):32-37.

9. Fonseca NR, Penna AF. Profile of the family caregiver caring for patients with sequels of cerebral vascular accident. Cien Saude Colet 2008; 13(4):1175-1180.

10. Laurent MCR, Durant D, Abarno CP. Papel do enfermeiro na assistência a pacientes pediátricos e adolescentes com fibrose cística no Hospital de Clínicas de Porto Alegre. Clin Biomed Res 2011; 31(2):233-237.

11. Tavares KO, Barros Carvalho MD, Pelloso SM. Dificuldades vivenciadas por mães de pessoas com fibrose cística. Texto Contexto Enferm 2014; 23(2):294-300.

12. Luz GDS, Barros Carvalho MD, da Silva MRS. O significado de uma organização de apoio aos portadores e familiares de fibrose cística na perspectiva das famílias. Texto Contexto Enferm 2011; 20(1):127-134.

13. Costa ASM, Britto MCA, Nobrega SM, Vasconcelos MGL, Lima LS. Vivências de familiares de crianças e adolescentes com fibrose cística. Rev Bras Crescimento Desenvolvimento Hum 2010; 20(2):217-227.
14. Bolla BA, Fulconi SN, Baltor MRR, Dupas G. Cuidado da criança com anomalia congênita: a experiência da família. Esc Anna Nery 2013; 17(2):284-290.

15. Santos ELR, Rocha MSC, Jesus LKA, Rezende MIRC, Machado AV, Andrade TS, Oliveira TVC, Marques DR, Santos DM, Sousa DS. Cuidados específicos de mães com filhos portadores de fibrose cística. Scire Salutis 2014; 4(1):17-25.

16. Abarno CP, Ribeiro NRR. Trajetória da família do paciente com fibrose cística até a sua morte. Rev Soc Bras Enferm Ped 2012; 12(2):113-121.

17. Manoel MF, Teston EF, Waidman MAP, Decesaro MN, Marcon SS. As relações familiares e o nível de sobrecarga do cuidador familiar. Esc Anna Nery 2013; 17(2):346-353.

18. Williams B, Mukhopadhyay S, Dowell J, Coyle J. From child to adult: an exploration of shifting family roles and responsibilities in managing physiotherapy for cystic fibrosis. Soc Sci Med 2007; 65(10):2135-2146.

19. Pizzignacco TM, de Lima RA. Socialization of children and adolescents with cystic fibrosis: support for nursing care. Rev Lat Am Enfermagem 2006; 14(4):569-577.

20. Pizzignacco TP, Mello DF, Lima RG. The experience of disease in cystic fibrosis: the paths to comprehensive care. Rev Esc Enferm USP 2011; 45(3):638-644.

21. Silva T, Schenkel EP, Mengue SS. Information level about drugs prescribed to ambulatory patients in a university hospital. Cad Saude Publica 2000; 16(2):449-455.

22. Müller CF, Backes E. A importância das associações de fibrose cística na vida dos pacientes e familiares. Clin Biomed Res 2011;31(2):123-124.

Artigo apresentado em 16/02/2016

Aprovado em 16/08/2016

Versão final apresentada em 18/08/2016 